\title{
Psychological Impact of COVID-19 on Frontline Healthcare Workers in Saudi Arabia
}

\author{
Sulafa Alqutub ${ }^{1}$, Mahmoud Mahmoud ${ }^{2}$, Tahani Baksh ${ }^{3}$ \\ 1. Family and Community Medicine, University of Jeddah, Jeddah, SAU 2. Family and Community Medicine, Imam \\ Muhammad Ibn Saud Islamic University, Riyadh, SAU 3. General Directorate, Ministry of Health, Jeddah, SAU
}

Corresponding author: Sulafa Alqutub, stalqutub@uj.edu.sa

\begin{abstract}
This study assesses the determinants and severity of psychological distress among frontline Ministry of Health workers within Saudi Arabia during the rapid acceleration phase of the coronavirus disease 2019 (COVID-19) epidemic. Moreover, we assess distress sustainability and stress-coping behaviors. We conducted an online national cross-sectional survey. The Kessler Psychological Distress Scale (k10) is a highly reliable instrument used to assess depression and anxiety. We evaluated stress-coping behavior and the persistence of the disorders. Binary logistic regression identified the sociodemographic factors related to severe distress.
\end{abstract}

The prevalence of severe psychological distress among COVID-19 frontline healthcare workers (HCWs) was 27.3\%. Factors associated with severe psychological distress in multiple regression analyses were male gender $(p<0.001)$, working for $>45$ hours/week $(p=0.009)$, age of $>40$ years $(p=0.038)$, years of experience for more than seven years $(p=0.048)$, Asir region $(p=0.003)$, and using psychological services $(p<0.001)$. The prevalence of severe psychological distress was $27.3 \%$. Factors associated with severe psychological distress in multiple regression analyses were male gender, working $>45$ hours/week, age, years of experience, region, and using psychological services. The results form a foundation for targeted psychological health support services at the individual and institutional levels to prevent progression to mental illness.

Review began 05/15/2021 Review ended 05/22/2021 Published 05/28/2021

\section{๑) Copyright 2021}

Alqutub et al. This is an open access article distributed under the terms of the Creative Commons Attribution License CC-BY 4.0., which permits unrestricted use, distribution, and reproduction in any medium, provided the original author and source are credited.
Categories: Preventive Medicine, Psychology, Public Health

Keywords: males, coping behavior, healthcare workers, kessler psychological distress scale, k10, covid 19

\section{Introduction}

Since the beginning of the coronavirus disease 2019 (COVID-19) pandemic and with the announcement of the first confirmed case in March 2020, the Saudi Ministry of Health (MOH) and Saudi Center of Disease Control (SCDC) took the lead in the battle against the disease. According to the latest statistics for 2019, the Saudi MOH oversees 286 hospitals or $57 \%$ of the hospitals in the Kingdom of Saudi Arabia (KSA). The total number of primary healthcare centers (PHCS) is 2261. During the 2020 pandemic, $20 \mathrm{MOH}$ hospitals were referral centers for COVID-19 cases across the nation, along with a variable number of quarantine facilities; these facilities dealt with suspected and/or confirmed cases of COVID-19 [1]

Frontline healthcare workers (HCWs) are workers in direct contact with suspected and/or confirmed cases within any previously mentioned facilities. Workers face considerable stress, anxiety, and depression. Hospital workers, especially in the emergency department, intensive care unit, and infectious disease ward, are at greater risk of anxiety, depression, and psychological disorders [2].

The Kessler psychological distress scale $(\mathrm{k} 10)$ is a psychometric instrument designed as a sensitive tool to discriminate cases of serious mental illness (SMI) from non-cases using a cutoff for the range of clinically significant distress. Australian and Canadian national health interview surveys use the k10. According to the Victorian population health survey, the k10 does not determine major mental illnesses such as psychosis.

The k10 uses 10 questions to measure a subject's psychological distress over the previous one-month period. The k10 has been validated as a simple measure of anxiety, depression, and worry, or what is generally called psychological distress [3].

The Arabic k10's reliability and validity were tested on Arabic speakers in different countries, including occupied Palestinian territories [4]. Arguments ensued about the k10 translation and culture adaptation and the difficulty of establishing cutoffs for all cultural groups in multicultural societies. However, recommendations say to use the $\mathrm{k} 10$ as a screening tool followed by a further clinical assessment to rule out mental illness [5].

During the COVID-19 epidemic in China, Kang et al. stated that protecting medical workers' mental health is crucial for controlling the epidemic and protecting their health [6]. The most recently published local study describing the psychological burden among nurses in Riyadh, KSA, concluded that practicing male 
This work aimed at measuring the severity of psychological distress and the contributing factors affecting the frontline moh workers, during the rapid escalation phase of the COVID-19 pandemic within KSA.

\section{Materials And Methods}

\section{Inclusion and exclusion criteria, instrument, data collection methods, and sites}

This cross-sectional survey included frontline HCWs at the Saudi MOH facilities. In addition to information on sociodemographic factors, job category, and years of experience, we used the K10. The Arabic version of the K10 was developed and used by the World Health Organization Composite International Diagnostic Interview Advisory Committee. The instrument is available in Arabic and English on the US National Comorbidity survey web page of the National Comorbidity Survey (NCS) [8]. The recently validated K10 version psychometric construct used its first six items to assess the severity of anxiety and its last four to assess the severity of depression. The anxiety scale included tiredness, nervousness, severe nervousness, restlessness, severe restlessness, and feelings toward the effort. The depression-scale items included hopelessness, depressed mood, the severity of depressive mood, and feelings of worthlessness. Each item within the $\mathrm{K} 10$ was based on a five-point scale from 0 (none of the time) to 5 (all of the time) and added for a total score ranging from 0 to 50 . Higher scores indicated higher levels of psychological distress [9].

The cutoff point scores for K10 to differentiate between nonspecific distress and cases with SMI were predetermined in the 2001 Victorian population health survey. The cutoff point for the scores was: 10-19 likely well, 20-24 mild disorder likely, 25-29 moderate disorder likely, and 30-50 severe disorder likely [3]. In this survey, we adopted the cutoff point scores from the Victorian population health survey.

Moreover, in addition to the K10, respondents who reported psychological distress in the past 30 days answered additional questions on the disorder's persistence and its impact on their health and ability to perform daily routines [8]. We assessed stress-coping skills through questions on exercise, meditation, and smoking habits. Questions on psychological or psychiatric help-seeking behavior over the last 12 months determined professional mental health service use in the past year [8].

The online data collection link remained open from the 20th through the 26th week of the 2020 epidemic, a period marked by a rapid acceleration in the epidemic curve (Figure 1) [10]. The MOH intranet portal sends the data collection link containing the Arabic and English language versions.

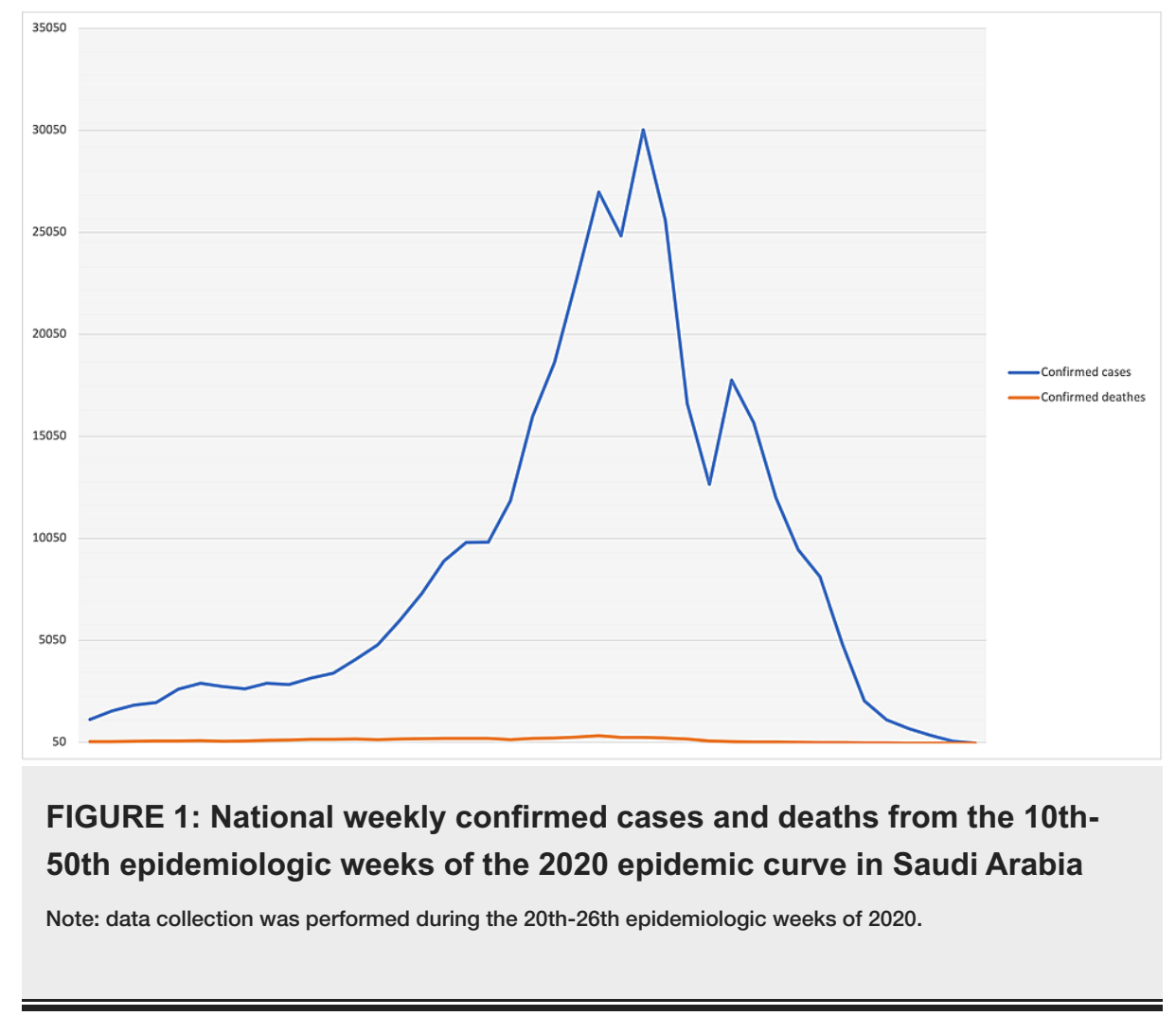

Sample size calculation and statistical analysis 
In 2016, a published study on psychological distress in Saudi Arabia among medical students using the K10 reported a psychological distress prevalence of 34\% [11]. The proportion of surveyed HCWs with psychological co-morbidities was roughly $35 \%$ based on a previous study of the severe acute respiratory syndrome coronavirus (SARS) outbreak [12]. A total of 228,171 HCWs, including Saudis and non-Saudis, are in the $\mathrm{MOH}$. The estimated number of frontline participants in the COVID-19 team is $30 \%$ of the total number of participants $(68,451)$. OpenEpi (www.OpenEpi.com) info was used, and the concluded sample size was 1901 at the $95 \%$ confidence interval with a margin of error of $2.1 \%$. We used the following equation in the sample size calculation.

$\mathrm{n}=\left[\operatorname{DEFF}^{*} \mathrm{~Np}(1-\mathrm{p})\right] /\left[\left(\mathrm{d} 2 / \mathrm{Z} 21-\alpha / 2 *(\mathrm{~N}-1)+\mathrm{p}^{*}(1-\mathrm{p})\right]\right.$. Where DEFF is the design effect, $\mathrm{p}^{*}$ is the estimated proportion.

For this analysis, the K10 score was divided into two groups: severe psychological disorder and nonsevere psychological disorder. HCWs with scores of 30-50 were part of the severe psychological disorder category, and HCWs with scores of 10-29 were part of the nonsevere psychological disorder category.

Frequency distributions were used to describe categorical variables. The mean and standard deviation reported central tendency measures. Differences between groups were analyzed using the chi-square test for categorical variables and the independent $t$-test for continuous outcome variables. Significance $(\mathrm{P})$ was set at 0.05. All the significant variables in the univariate analysis were entered into a multiple regression model to identify the independent predictors of severe psychological distress. Analysis was performed with the Statistical Package for the Social Sciences (SPSS) (IBM Corp., Version 21.0. Armonk, NY).

The K10’s reliability was tested using Cronbach's alpha.

\section{Results}

\section{Sociodemographic and work-related characteristics}

Two-thousand ninety-four $(2,094)$ of 2,499 HCWs agreed to participate (response rate $=81 \%$ ). The majority were male (65.2\%), Saudi (87.6\%), under 40 years old (74.3\%), married (77.4\%), with a monthly income of more than 10,000 SAR. Sixty-two (3.0\%) of the frontline workers reported a history of COVID-19 diagnosis (Table 1). 


\section{Cureus}

n

Gender

Female 728 34.8

Male

1366

Nationality

Saudi

Non-Saudi

1835

87.6

259

Age group

$<40$

$\geq 40$

Marital status

Married

1621

77.4

Singles

379

18.1

Widowed/divorced

Income

$\leq 10000$

749

$>10000$

1345

COVID-19 infection

Yes

No

1395

66.6

I don't know

637

Smoking

Yes

No

Exercise

Yes

656

No

1438

68.7

Mediation

Yes

No

1568

Visiting psychiatric clinic last year

At least one time

No

1923

Try to reach psychological services last year

At least one time 


\section{Cureus}

Nurses formed the majority of the sample (47.9\%), followed by other health specialties (emergency medical services technicians, psychologists, and social worker staff) (26.45\%) and doctors (14.7\%). Most were hospital frontline workers (63\%), followed by primary HCWs (24\%), and the smallest proportion was from quarantine facilities (14\%) (Table 2).

n

Work site

Hospital

Primary health care center

Quarantine

Years of experience

$\leq 7$

$>7$

Working hours/week

$\leq 45$

$>45$

Health care worker category

Doctors

304

Nurses

Administrative

991

227

572

Region of practice

Medina

Najran

North boarders (Hail, Arar, \& Aljouf)

Asir (Abha \& Albaha)

Eastern province

Riyadh

Qassim

Makkah (Jeddah, Taif, \& Alqunfudah)

Hafralbaten

Other
14.7

27.3

$\%$

62.5

13.9

25.9

74.1

30.1

69.9

47.9

11.0

3.8

15.2

9.4

10.6

5.6

29.9

4.9

16.2

3.4

1.0

TABLE 2: Work-related characteristics of the participants $(n=2094)$

\section{Coping skills and help-seeking behavior}

Approximately $31.3 \%$ exercised regularly, 29.5\% were smokers, and $25.1 \%$ reported practicing meditation. Regarding past-year professional mental health service utilization, $8.2 \%$ visited the psychiatry clinic at least once over the last year and $6.5 \%$ tried to reach psychological services at least once over the previous year.

\section{Perceived sustainability of the stress}

Approximately one-quarter of the participants believed that psychological distress was always related to any of the health conditions they may have suffered over the last month (Table 3). Despite the prevalence of psychological distress, about $60 \%$ denied their inability to complete either half or all their daily tasks. 


\section{Cureus}

Regarding the reported daily inability to complete either half or all their daily tasks, the proportion did not exceed $6 \%$ of the total responses.

Response

A little of the time

Some of the time

Most of the time

All the time n (\%)

391 (19)

205 (10)

1013 (49)

480 (23)

TABLE 3: The perceived stress-related health conditions over the last month

\section{Level of psychological distress among COVID-19 frontline HCWs}

The mean (SD) K10 score was 23.1 (+21). Severe psychological distress was reported by $\mathrm{n}=570$ (27.3\%) of the participants while mild and moderate psychological distress was reported by $n=328(15.7 \%)$ and $n=277$ (13.2\%), respectively.

\section{Factors associated with severe psychological distress in univariate analysis}

Psychological distress was significantly higher among females $(p<0.001)$, aged $>40$ years $(p=0.001)$, married $(\mathrm{p}=0.045)$, and those who did not reach psychological services last year $(\mathrm{p}=0.048)$. Regarding workrelated characteristics, psychological distress was significantly higher among those who worked $>45$ hours per week $(p=0.010)$ and those who had more than seven years of experience $(p=0.007)$. There was an overall significant association between psychological distress and practice region, where the highest reported severe distress was in Asir and the lowest was in the Hafralbaten area $(\mathrm{p}=0.005)$ (Table 4). 


\section{Cureus}

\begin{tabular}{|c|c|c|}
\hline & Severe psychological distress $\mathrm{n}(\%)$ & P-value \\
\hline \multicolumn{3}{|l|}{ Gender } \\
\hline Female & $157(21.7)$ & \\
\hline Male & $413(30.3)$ & $<0.001$ \\
\hline \multicolumn{3}{|l|}{ Nationality } \\
\hline Saudi & $504(27.5)$ & \\
\hline Non-Saudi & & 0.589 \\
\hline \multicolumn{3}{|l|}{ Age group } \\
\hline$<40$ & $395(25.4)$ & \\
\hline$\geq 40$ & $1 / 6(32.7)$ & 0.001 \\
\hline \multicolumn{3}{|l|}{ Marital status } \\
\hline Married & $23(24.5)$ & \\
\hline Singles & 463(28.6) & \\
\hline Widowed/divorced & $85(22.4)$ & 0.045 \\
\hline \multicolumn{3}{|l|}{ Income } \\
\hline$\leq 10000$ & $186(24.8)$ & \\
\hline$>10000$ & $385(28.6)$ & 0.062 \\
\hline \multicolumn{3}{|l|}{ COVID-19 infection } \\
\hline Yes & $21(33.9)$ & \\
\hline No/l don't know & $550(27.1)$ & 0.236 \\
\hline \multicolumn{3}{|l|}{ Smoking } \\
\hline Yes & $171(27.7)$ & \\
\hline No & $400(27.1)$ & 0.789 \\
\hline \multicolumn{3}{|l|}{ Exercise } \\
\hline Yes & $196(29.9)$ & \\
\hline No & $375(26.1)$ & 0.070 \\
\hline \multicolumn{3}{|l|}{ Mediation } \\
\hline Yes & $151(28.7)$ & \\
\hline No & $420(26.8)$ & 0.392 \\
\hline \multicolumn{3}{|c|}{ Visiting psychiatric clinic last year } \\
\hline At least one time & $53(31.7)$ & \\
\hline No & $518(26.9)$ & 0.177 \\
\hline \multicolumn{3}{|c|}{ Try to reach psychological services last year } \\
\hline At least one time & $4 /(26.8)$ & \\
\hline No & $524(34.6)$ & 0.048 \\
\hline
\end{tabular}

TABLE 4: Association between severe psychological distress and sociodemographic variables in univariate analysis $(n=2094)$ 


\section{Cureus}

\section{Factors associated with severe psychological distress in multivariate}

analysis

The identified predictors included male gender $(\mathrm{OR}=1.5,95 \% \mathrm{CI} 1.21-1.90)$, over 40 years old $(\mathrm{OR}=1.3$, 95\%CI 1.01-1.60), working more than 45 hours per week $(\mathrm{OR}=1.3,95 \% \mathrm{CI} 1.07-1.64)$, with more than seven years of experience (OR $=1.2,95 \% \mathrm{CI} 1.23-1.53)$. Furthermore, those who did not try to reach psychological services last year had a higher risk of psychological distress ( $\mathrm{OR}=1.5,95 \% \mathrm{CI} 1.03-2.18)$. As to regional differences, those who worked in Asir had higher distress than those who worked in Hafralbaten $(\mathrm{OR}=2.6$, 95\%CI 1.38-5.05) (Tables 5-6).

\begin{tabular}{|c|c|c|}
\hline & Severe psychological distress $\mathrm{n}(\%)$ & P-value \\
\hline \multicolumn{3}{|l|}{ Worksite } \\
\hline Hospital & $359(27.4)$ & \\
\hline Primary health care center & $125(25.4)$ & \\
\hline Quarantine & 87(29.8) & 0.393 \\
\hline \multicolumn{3}{|l|}{ Years of experience } \\
\hline$\leq 7$ & 124(22.8) & \\
\hline$>7$ & $447(28.8)$ & 0.007 \\
\hline \multicolumn{3}{|l|}{ Working hours/week } \\
\hline$\leq 45$ & 196(25.6) & \\
\hline$>45$ & $375(31.1)$ & 0.010 \\
\hline \multicolumn{3}{|l|}{ Healthcare worker category } \\
\hline Doctors & $84(27.6)$ & \\
\hline Nurses & $282(28.5)$ & \\
\hline Administrative & $51(22.5)$ & \\
\hline Other & $140(25.6)$ & 0.261 \\
\hline \multicolumn{3}{|l|}{ Region of practice } \\
\hline Medina & $23(28.8)$ & \\
\hline Najran & $82(25.9)$ & \\
\hline North boarders (Hail, Arar, \& Aljouf) & $48(24.6)$ & \\
\hline Asir (Abha \& Albaha) & $87(39.5)$ & \\
\hline Eastern province & $31(26.7)$ & \\
\hline Riyadh & $164(26.4)$ & \\
\hline Qassim & 34 (33.3) & \\
\hline Makkah (Jeddah, Taif, \& Alqunfudah) & $81(24.1)$ & \\
\hline Hafralbaten & $14(19.7)$ & \\
\hline Other & $5(23.8)$ & 0.005 \\
\hline
\end{tabular}

TABLE 5: Association between severe psychological distress and work-related variables in univariate analysis $(n=570)$ 


\begin{tabular}{|c|c|c|c|c|c|}
\hline & $\mathrm{B}$ & S.E. & P-value & Odds Ratıo & $95.0 \% \mathrm{Cl}$ \\
\hline Males & 0.420 & 0.113 & 0.000 & 1.5 & $1.21-1.90$ \\
\hline Age $\geq 40$ years & 0.243 & 0.117 & 0.038 & 1.3 & $1.01-1.60$ \\
\hline Working hours/week (>45) & 0.284 & 0.108 & 0.009 & 1.3 & $1.07-1.64$ \\
\hline Try to reach psychological services last year (no vs yes) & 0.406 & 0.192 & 0.034 & 1.5 & 1.03-2.18 \\
\hline Years of experience (>7 years) & 0.182 & 0.126 & 0.048 & 1.2 & $1.23-1.53$ \\
\hline Asir (reference= Hafralbaten) & $0.9 / 2$ & 0.331 & 0.003 & 2.6 & $1.38-5.05$ \\
\hline Constant & -2.092 & 0.330 & 0.000 & 0.12 & $1.21-1.90$ \\
\hline
\end{tabular}

TABLE 6: Factors associated with severe psychological distress in multivariate analysis

\section{Discussion}

Our study showed that the overall national prevalence of severe psychological distress among all COVID-19 frontline HCWs was $27.3 \%$. Subgroup analysis showed severe psychological distress among male frontline workers. Males suffered a more significant impact of psychological distress than female frontline workers $(\mathrm{p}$ $=.001$ ). Within this context, a recent review by Bohlken, Schömig, Lemke, Pumberger, and Riedel-Heller on 14 different studies about psychological distress in COVID-19 HCWs reported that the prevalence of psychological distress ranged from $2.2 \%$ to $14.5 \%$ of all participants [13]. In an extreme finding published by Lai et al. in 2020, nurses, women, frontline HCWs, and those working in Wuhan, China, reported severe degrees of psychological distress, with a prevalence of $71 \%$ [12]. The sex differences observed in our study were attributed to the higher representation of male workers, forming $65 \%$ of the sample. Moreover, the observed variability in the prevalence of psychological distress across various studies globally can be related to the difference in psychometric tools used and male roles in Middle Eastern society.

Our findings align with those of Balay-Odao et al., who performed a study in Riyadh and concluded that male Saudi national frontline nurses providing direct care for COVID-19 patients were impacted significantly by psychological burden [7]. Moreover, male nurses had a higher mean psychological burden score than female nurses during the 2020 pandemic. The research findings were based on the role of males within Asian families. Likewise, male workers are usually the primary source of income and are the primary providers for the family.

Our study showed a $27.6 \%$ prevalence $(n=84)$ of severe psychological distress among frontline doctors, as shown in Table 6. The findings contradict Almater, Tobaigy, Younis, Alaqeel, and Abouammoh among 107 ophthalmologists in Riyadh, Saudi Arabia, who showed a prevalence of severe depression and anxiety of $3.7 \%$ and $5.6 \%$, respectively [14]. The observed higher severity might be due to the broad scope of practice observed for our participating physicians. However, the differences in psychological distress severity among physicians in different specialties at the national level should be assessed in further studies using consistent standardized psychometric tools.

Frontline HCWs working within the KSA who worked for more than 45 hours a week were at the highest risk of severe psychological distress. Several studies have concluded the association between depression and mental distress with long-term weekly working hours [15-16]. However, the results were contradictory based on the country of practice, type of occupation, and working conditions. In the previous study on the average weekly working hours, Kim et al. concluded that for unsecured workers, 41 to 52 hours of working per week correlates with a lower risk of depression and anxiety than working for less than 41 hours per week [15]. From the perspective of COVID-19, a recent study reported by Cai, Tu, Ma, Chen, Fu, Jiang, and Zhuang from Hubei, China, concluded that exhaustion and long working hours during COVID-19 were significantly associated with stress in frontline HCWs [17]. The relation between working hours and occupational health issues is being investigated at the organizational and national levels to resolve the contradictory findings in various studies.

The reported rate of severe psychological distress was directly related to HCW age. At the ages of less than 40 years, the proportion of severe distress was $n=395(25.4 \%)$. Whereas, over age 40 , it increased to $n=176$ (32.7\%). Despite not asking the participants direct questions regarding concerns about infecting family members, more than three-quarters of the participants $(n=1621 ; 77.4 \%)$ were either married with children or married with no children (Table 1). Similarly, Cai et al. concluded that COVID-19 HCWs aged 31-40 years were stressed due to worries about infecting family members [17].

In all of the previously mentioned demographics, male workers, workers who were married, workers aged 
$>40$ years, workers who worked for more than 45 hours, workers with seven years of experience, and workers in the Asir regions were likely to be stressed because of the perceived risk of infecting family members. Studies have shown that workload, a history of previous infections, working in isolation words, exposure to and contact with severe cases, and the deaths of other HCWs were each identified as factors contributing to psychological distress [12,18-19].

This study also showed that workers at PHCs were less likely to suffer from severe psychological distress. This result is likely associated with the observed lower infection rates among PHC workers as compared to the $63 \%$ reported infection rate among hospital workers. Our findings can be due to exposure to mild cases in PHCs compared to exposure to more severe COVID-19 cases or deaths encountered in hospitals.

Most of the frontline HCWs who reported a high proportion of severe psychological distress denied trying to reach out to any psychological support services $(n=524 ; 34.6)$ within the last year. The findings support the notion of the absence of pre-existing psychological distress and/or illnesses. Unironically, the results from a recent study conducted by Ali, Cole, Ahmed, Hamasha, and Panos, in Alabama in the USA found that none of the participating nurses reported ever seeking help from a psychologist as a coping strategy during the current pandemic [20].

Our study found less frequent coping skills, such as exercising and meditation, at $31.3 \%$ and $25.5 \%$, respectively. Coping is one of the strategies used to mitigate the impact of stress. A recent study by Shechter, Diaz, Moise, Anstey, Ye, Agarwal, Abdalla, Brodie, Cannone, Chang, Claassen, Cornelius, Derby, Dong, Givens, Hochman, Homma, Kronish, Lee, Manzano, Mayer, McMurry, Moitra, Pham, Rabbani, Rivera, Schwartz, Schwartz, Shapiro, Shaw, Sullivan, Vose, Wasson, Edmondson, and Abdalla reported that physical activity/exercise was the most common coping behavior performed by New York HCWs during the COVID-19 pandemic (59\%). Yoga and meditation were employed [18]. They also reported access to psychotherapy services and online self-guided counseling use by 33\% of the participating HCWs [21]. Coping strategies, such as HCW training on building resilience to stress, were considered primary prevention by Preti et al. [22].

Our study findings on the low prevalence of physical activity among HCWs can be due to the low physical activity levels within the Saudi community. A systematic review conducted by Al-Hazzaa in 2018 reported that youth and adults are not active enough to meet the recommended guidelines for physical activity [23]. Moreover, in the Al-Jouf region, Banday, Want, Alris, Alrayes, and Alenzi concluded that 35\% of PHC physicians were physically inactive [24].

Smoking was a negative coping skill reported by $30 \%$ of frontline workers. The lack of physical activity and nicotine dependence should be addressed through public health programs to prevent the long-term effects of sedentary life and substance dependence. Recommended interventions include access to resilience training and online self-help psychological support services to prevent the progression of distress into severe mental illnesses [21-22].

Our findings yield results on the attributes of severe psychological distress encountered by MOH HCWs with COVID-19 within Saudi Arabia. The Kessler psychological distress scale (k10) for measuring anxiety and depression is a reliable scale used in many international public surveys. At the Saudi national level, our study is the first of its kind to assess the prevalence of psychological distress in an occupational setting. Also, none of the previous studies of this kind have included community (primary healthcare and quarantine) and hospital frontline health workers. Multivariate analysis for a relatively large sample has provided adjustment for the potential confounders in this kind of survey. Hence, we may draw a valid conclusion. Moreover, the findings will help identify policy interventions and develop preventive programs. Additionally, the results will help develop coping strategies at both individual and organizational levels.

This study is not a longitudinal cohort study; therefore, there are some limitations such as the risk of missing and/or reporting pre-existing mental health conditions. Additionally, despite a response rate of $81 \%$, response bias may still exist because non-responders were overwhelmed and/or distressed and may not have been interested in filling out the survey. Regional differences and organizational work-related potential stressors, such as workload, payment status, and job satisfaction, were not assessed and are beyond the scope of this study.

\section{Conclusions}

Self-rated severe psychological distress without mental disorder is significantly prevalent among male COVID-19 frontline workers aged $>40$ years in Saudi Arabia. However, distress did not affect their daily life routines. Hospital frontline workers with a history of infection with severe acute respiratory syndrome coronavirus 2 (SARS-CoV-2) were more likely to report severe psychological distress. Additionally, working an average of more than 45 hours per week was a strong predictor of severe psychological distress. Severe distress was perceived to be highly related to their health conditions and suffering over the last month before responding to the survey.

\section{Additional Information}




\section{Disclosures}

Human subjects: Consent was obtained or waived by all participants in this study. MOH Central Institutional Review Board issued approval No. 20-\#634M. Animal subjects: All authors have confirmed that this study did not involve animal subjects or tissue. Conflicts of interest: In compliance with the ICMJE uniform disclosure form, all authors declare the following: Payment/services info: The author(s) disclosed receipt of the following financial support for the research, authorship, and/or publication of this article: This work was supported by the General directorate of research, MOH, KSA [20-\#634M] for COVID19 grants. . Financial relationships: All authors have declared that they have no financial relationships at present or within the previous three years with any organizations that might have an interest in the submitted work. Other relationships: All authors have declared that there are no other relationships or activities that could appear to have influenced the submitted work.

\section{Acknowledgements}

We thank the Director-General of Internal Communication, Mrs. Salwa AlEmran, and Mrs. Shaza Alshareef for their valuable contributions and support in facilitating the process of data collection as planned. Sincere thanks to Dr. Aldabi Sami for his support in results analysis. Finally, we thank the frontline HCWs for their time in completing the questionnaire despite unprecedented worksite challenges.

\section{References}

1. MOH statistics and indicators. (2019). Accessed: March 24, 2021: https://www.moh.gov.sa/en/Ministry/Statistics/Pages/default.aspx.

2. Shreffler J, Petrey J, Huecker M: The impact of COVID-19 on healthcare worker wellness: a scoping review . West J Emerg Med. 2020, 21:1059-66. 10.5811/westjem.2020.7.48684

3. Victoria State Government. Victorian population health survey 2001. Selected findings . (2001). Accessed: May 10, 2021: https://www2.health.vic.gov.au/about/publications/policiesandguidelines/Victorianpopulation-health-survey-2001-Selec....

4. Easton SD, Safadi NS, Wang Y, Hasson RG 3rd: The Kessler psychological distress scale: translation and validation of an Arabic version. Health Qual Life Outcomes. 2017, 15:215. 10.1186/s12955-017-0783-9

5. Stolk Y, Kaplan I, Szwarc J: Clinical use of the Kessler psychological distress scales with culturally diverse groups. Int J Methods Psychiatr Res. 2014, 23:161-83. 10.1002/mpr.1426

6. Kang L, Li Y, Hu S, et al.: The mental health of medical workers in Wuhan, China dealing with the 2019 novel coronavirus. Lancet Psychiatry. 2020, 7 :e14. 10.1016/S2215-0366(20)30047-X

7. Balay-Odao EM, Alquwez N, Inocian EP, Alotaibi RS: Hospital preparedness, resilience, and psychological burden among clinical nurses in addressing the COVID-19 crisis in Riyadh, Saudi Arabia. Front Public Health. 2020, 8:573932. 10.3389/fpubh.2020.573932

8. National Comorbidity Survey (NCS). (2020). Accessed: March 24.2021: https://www.hcp.med.harvard.edu/ncs/.

9. Sampasa-Kanyinga H, Zamorski MA, Colman I: The psychometric properties of the 10-item Kessler Psychological Distress Scale (K10) in Canadian military personnel. PLoS One. 2018, 13:e0196562. 10.1371/journal.pone.0196562

10. European Centre for Disease Prevention and Control: 2020. Download historical data on the daily number of new reported COVID-19 cases and deaths worldwide. (2020). Accessed: December 14.2020: https://www.ecdc.europa.eu/en/covid-19.

11. Saeed AA, Bahnassy AA, Al-Hamdan NA, Almudhaibery FS, Alyahya AZ: Perceived stress and associated factors among medical students. J Family Community Med. 2016, 23:166-71. 10.4103/2230-8229.189132

12. Lai J, Ma S, Wang Y, et al.: Factors associated with mental health outcomes among health care workers exposed to coronavirus disease 2019. JAMA Netw Open. 2020, 3:e203976. 10.1001/jamanetworkopen.2020.3976

13. Bohlken J, Schömig F, Lemke MR, Pumberger M, Riedel-Heller SG: COVID-19 pandemic: stress experience of healthcare workers - a short current review [Article in German]. Psychiatr Prax. 2020, 47:190-7. 10.1055/a-1159-5551

14. Almater AI, Tobaigy MF, Younis AS, Alaqeel MK, Abouammoh MA: Effect of 2019 coronavirus pandemic on ophthalmologists practicing in Saudi Arabia: a psychological health assessment. Middle East Afr J Ophthalmol. 2020, 27:79-85. 10.4103/meajo.MEAJO_220_20

15. Kim W, Park EC, Lee TH, Kim TH: Effect of working hours and precarious employment on depressive symptoms in South Korean employees: a longitudinal study. Occup Environ Med. 2016, 73:816-22. 10.1136/oemed-2016-103553

16. Wong K, Chan AHS, Ngan SC: The effect of long working hours and overtime on occupational health: a meta-analysis of evidence from 1998 to 2018. Int J Environ Res Public Health. 2019, 16:2102. 10.3390/ijerph16122102

17. Cai H, Tu B, Ma J, Chen L, Fu L, Jiang Y, Zhuang Q: Psychological impact and coping strategies of frontline medical staff in Hunan between January and March 2020 during the outbreak of coronavirus disease 2019 (COVID-19) in Hubei, China. Med Sci Monit. 2020, 26:e924171. 10.12659/MSM.924171

18. El-Hage W, Hingray C, Lemogne C, et al.: Health professionals facing the coronavirus disease 2019 (COVID19) pandemic: what are the mental health risks? [Article in French]. Encéphale. 2020, 46:73-80.

19. Chung JPY, Yeung WS.: Staff mental health self-assessment during the COVID-19 outbreak. East Asian Arch Psychiatry. 2020, 30:34-10. 10.3316/INFORMIT.099574617030273

20. Ali H, Cole A, Ahmed A, Hamasha S, Panos G: Major stressors and coping strategies of frontline nursing staff during the outbreak of coronavirus disease 2020 (COVID-19) in Alabama. J Multidiscip Healthc. 2020, 13:2057-68. 10.2147/JMDH.S285933 


\section{Cureus}

21. Shechter A, Diaz F, Moise N, et al.: Psychological distress, coping behaviors, and preferences for support among New York healthcare workers during the COVID-19 pandemic. Gen Hosp Psychiatry. 2020, 66:1-8. 10.1016/i.genhosppsych.2020.06.007

22. Preti E, Di Mattei V, Perego G, et al.: The psychological impact of epidemic and pandemic outbreaks on healthcare workers: rapid review of the evidence. Curr Psychiatry Rep. 2020, 22:43. 10.1007/s11920-02001166-z

23. Al-Hazzaa HM: Physical inactivity in Saudi Arabia revisited: a systematic review of inactivity prevalence and perceived barriers to active living. Int J Health Sci (Qassim). 2018, 12:50-64.

24. Banday AH, Want FA, Alris FF, Alrayes MF, Alenzi MJ: A cross-sectional study on the prevalence of physical activity among primary health care physicians in Aljouf region of Saudi Arabia. Mater Sociomed. 2015, 27:263-6. 10.5455/msm.2015.27.263-266 